\title{
Extraction du Gallium de l'Indium et de Quelques Autres Métaux en Milieu Thiocyanate par le Dibutylphénacylphosphonate (HDBPP)
}

\author{
J. P. BRUNETTE, ${ }^{a}$ M. J. F. LEROY, ${ }^{a}$ B. CECCAROLI ${ }^{b}$ et J. ALSTAD ${ }^{b}$
}

${ }^{a}$ Laboratoire de Chimie Minérale, Ecole Nationale Supèrieure de Chimie de Strasbourg, Université Louis Pasteur, 1, rue Blaise Pascal, 67008 Strasbourg Cedex, France et ${ }^{\mathrm{b}}$ Section of Nuclear Chemistry, Department of Chemistry, University of Oslo, Oslo 3, Norway

The extraction of gallium(III), indium(III), iron(III), vanadium(IV) and some other metals from aqueous thiocyanate media by dibutylphenacylphosphonate (HDBPP) in toluene has been studied. $\mathrm{Ga}(\mathrm{NCS})_{3}$ $(\mathrm{HDBPP})_{3}, \quad \mathrm{In}(\mathrm{NCS})_{3}(\mathrm{HDBPP})_{3} \quad \mathrm{Fe}(\mathrm{NCS})_{3}$ $(\mathrm{HDBPP})_{3}$ and $\mathrm{VO}(\mathrm{NCS})_{2}(\mathrm{HDBPP})_{3}$, together with the thiocyanic acid, are extracted. HDBPP is bonded to the extracted metals only through one atom, probably the oxygen of the $\mathrm{P}=\mathrm{O}$ group.

Les extractions du gallium(III), de l'indium(III), du fer(III), du vanadium(IV) et de quelques autres métaux en milieu thiocyanate par le dibutylphénacylphosphonate (HDBPP) dans le toluène ont été étudiées. Les espèces $\mathrm{Ga}(\mathrm{NCS})_{3}(\mathrm{HDBPP})_{3}$, In$(\mathrm{NCS})_{3}(\mathrm{HDBPP})_{3}, \mathrm{Fe}(\mathrm{NCS})_{3}(\mathrm{HDBPP})_{3}$ et VO$(\mathrm{NCS})_{2}(\mathrm{HDBPP})_{3}$, ainsi que l'acide thiocyanique, sont extraits. Le HDBPP n'est lié aux métaux extraits que par l'intermédaire d'un seul atome vraisemblablement l'oxygène du groupement $\mathrm{P}=\mathrm{O}$.

De nombreuses publications sur l'extraction des métaux en milieu thiocyanate ont paru ces dernières années: Sekine, Hasegawa et coll., ${ }^{1-7}$ Specker et coll., ${ }^{8,9}$ Golub et coll. ${ }^{10}$ et Khopkar et coll., ${ }^{11}$ en particulier, ont étudié l'extraction des terres rares, de In, $\mathrm{Ga}, \mathrm{Zn}, \mathrm{Hg}, \mathrm{Be} .$. par des extractants neutres tels que le phosphate de tributyle, l'oxyde de trioctylphosphine et la méthylisobutylcétone. D'autres auteurs ont utilisé des extractants échangeurs d'anions: Mikheev et coll. ont étudié l'extraction du gallium par la tri-n-octylamine ${ }^{12}$ et nous nous sommes intéressés à la séparation de l'aluminium, du gallium et de l'indium par la trilaurylamine. ${ }^{13}$
Certaines études ont finalement conduit à des applications. ${ }^{14,15}$

Le travail présenté ici concerne essentiellement l'extraction du gallium, de l'indium et de quelques autres métaux en milieu thiocyanate par le dibutylphénacylphosphonate $(\mathrm{BuO})_{2} \mathrm{P}(\mathrm{O}) \mathrm{CH}_{2} \mathrm{C}(\mathrm{O}) \mathrm{C}_{6} \mathrm{H}_{5}$, noté HDBPP, en solution dans le toluène. Cela fait partie d'une étude plus générale des propriétés extractives des $\beta$-cétophosphonates. De tels composés sont à priori intéressants du fait de la dissymétrie du pouvoir donneur des deux groupements fonctionnels $\mathrm{C}=\mathrm{O}$ et $\mathrm{P}=\mathrm{O}$ qui peut conduire à une grande sélectivité, et de la présence d'un groupe méthylène à hydrogène mobile qui en fait potentiellement des chélatants acides asymétriques. Peu d'études concernant l'utilisation des $\beta$-cétophosphonates comme extractants ont été publiées à ce jour. Nous citerons les travaux de Meider-Gorican et coll. ${ }^{16-17}$ concernant l'extraction des couples de métaux $\mathrm{Zr}-\mathrm{Hf}$ et $\mathrm{Nb}-\mathrm{Ta}$ ainsi que nos propres travaux qui ont porté essentiellement sur l'extraction de l'uranium. ${ }^{18}$

\section{PARTIE EXPERIMENTALE}

Phases organiques. Ce sont des solutions de HDBPP dans du toluène "Fluka purum" à des concentrations telles que $0,01 \mathrm{M} \leq[\mathrm{HDBPP}] \leq 0,4 \mathrm{M}$. Le HDBPP a été préparé suivant la méthode décrite précèdemment ${ }^{18}$ qui fait intervenir la réaction de la bromoacétophénone sur le phosphite de tributyle. 
Phases aqueuses. Elles sont obtenues par attaque de $1 \mathrm{~g}$ de métal par $15 \mathrm{ml}$ d'acide nitrique concentré ou par disșolution aqueuse de son équivalent en nitrate. La solution est ensuite diluée; la concentration en $\mathrm{KSCN}$ et le $\mathrm{pH}$ de la solution sont ajustés par addition de solutions de $\mathrm{KSCN}$ ou de $\mathrm{HNO}_{3}$ concentrées. Dans le cas du vanadium nous avons dissous du sulfate de vanadyle dans de l'eau acidifiée par $\mathrm{H}_{2} \mathrm{SO}_{4}$; l'utilisation de métavanadate d'ammonium est également possible du fait de la réduction qui se fait en présence d'ions thiocyanates. (concentrations métalliques utilisées lors des extractions: $\mathrm{Al}=200 \mathrm{mg} / \mathrm{l} \mathrm{Ga}=200 \mathrm{mg} / \mathrm{l} ; \mathrm{In}=500 \mathrm{mg} / \mathrm{l} ; \mathrm{Zn}=$ $250 \mathrm{mg} / \mathrm{l} ; \mathrm{Cd}=200 \mathrm{mg} / \mathrm{l} ; \mathrm{Fe}=100 \mathrm{mg} / \mathrm{l}$ et $\mathrm{V}=250$ $\mathrm{mg} / \mathrm{l})$.

Extractions. Elles ont été réalisées par agitations manuelles de volumes égaux des deux phases pendant cinq minutes à une température de $19 \pm 1^{\circ} \mathrm{C}$. Les $\mathrm{pH}$ des solutions aqueuses à l'équilibre ont été mesurés à l'aide d'un $\mathrm{pH}$ mètre digital Philips $\mathrm{PW}$ 9408 équipé d'une électrode de verre Ingold 40I.88 NS K19. Un spectrophotomètre d'absorption atomique IL 453 a été utilisé pour la mesure des concentrations métalliques dans les deux phases. Dans le cas du vanadium, c'est la colorimétrie de l'ion peroxyvanadyle $\left[\mathrm{VO}\left(\mathrm{O}_{2}\right)\right]^{+}$a $460 \mathrm{~nm}$ qui a été choisie comme méthode de dosage.

Dosage de HSCN. Afin de doser l'acide thiocyanique extrait en phase organique ce dernier est réextrait par plusieurs lavages successifs de $5 \mathrm{ml} \mathrm{de}$ solution organique par une solution aqueuse de soude molaire. La solution de lavage est recueillie quantitativement, acidifiée par $\mathrm{HNO}_{3}$ puis dosée par $\mathrm{AgNO}_{3}$ 0,01 M. Le point d'équivalence est déterminé par potentiométrie.

\section{RESULTATS - DISCUSSION}

\section{Généralités}

En milieu thiocyanate, la plupart des métaux sont susceptibles de se complexer suivant les équilibres:

$$
\begin{aligned}
& \mathrm{M}^{m+}+n \mathrm{SCN}^{-} \stackrel{\beta_{n}}{=} \mathrm{M}(\mathrm{NCS})_{n}^{m-n} \\
& \beta_{n}=\frac{\left[\mathrm{M}(\mathrm{NCS})_{n}^{m-n}\right]}{\left[\mathrm{M}^{m+}\right]\left[\mathrm{SCN}^{-}\right]^{n}}
\end{aligned}
$$

L'agitation d'une telle solution aqueuse avec un extractant neutre $\mathrm{S}$ dissous dans un solvant organique peut faire passer le métal $M$ d'une phase à l'autre suivant l'équilibre:

$$
\begin{aligned}
& \mathrm{M}^{m+}+m \mathrm{SCN}^{-}+x \overline{\mathrm{S}} \stackrel{K}{\rightleftharpoons} \overline{\mathrm{M}(\mathrm{NCS})_{m} \mathrm{~S}_{x}} \\
& \mathrm{~K}=\frac{\left[\overline{\mathrm{M}(\mathrm{NCS})_{m} \mathrm{~S}_{x}}\right]}{\left[\mathrm{M}^{m+}\right]\left[\mathrm{SCN}^{-}\right]^{m}[\overline{\mathrm{S}}]^{x}}
\end{aligned}
$$

(les espèces de la phase organique sont surlignées)

Si l'on suppose que $x$ ne puisse prendre qu'une seule valeur, le coefficient de distribution $D_{\mathrm{M}}$ du métal $\mathrm{M}$ s'écrit alors:

$$
D_{\mathrm{M}}=\frac{\bar{C}_{\mathrm{M}}}{C_{\mathrm{M}}}=\frac{\left[\overline{\mathrm{M}(\mathrm{NCS})_{m} \mathrm{~S}_{x}}\right]}{\Sigma\left[\mathrm{M}(\mathrm{NCS})_{n}^{m-n}\right]}
$$

ce qui entraîne, en tenant compte des équations (1) et (2)

$D_{\mathrm{M}}=\frac{K\left[\mathrm{SCN}^{-}\right]^{m}[\overline{\mathrm{S}}]^{x}}{\sum_{n} \beta_{n}\left[\mathrm{SCN}^{-}\right]^{n}}$

soit, pour $\left[\mathrm{SCN}^{-}\right]$constant

$\log D_{\mathrm{M}}=\mathrm{cte}+x \log [\overline{\mathrm{S}}]$

Si le HDBPP se comporte vis-à-vis des métaux en milieu thiocyanate comme un simple extractant neutre, les courbes $\log D_{\mathrm{M}}=f(\log [\mathrm{HDBPP}])$, établies à diverses concentrations en ions $\mathrm{SCN}^{-}$ constantes, seront donc des droites de pentes égales au nombre de molécules de HDBPP fixées sur chaque atome de métal extrait. Avant d'étudier de telles courbes, nous avons évalué l'importance de l'extraction d'acide thiocyanique par le HDBPP dans les conditions d'extraction des métaux.

\section{Extraction de l'acide thiocyanique par le HDBPP}

Les extractions ont été réalisées à partir de solutions aqueuses molaires en $\mathrm{KSCN}$ en faisant varier le $\mathrm{pH}$ et la concentration en HDBPP de la phase organique. Soit $D_{\mathrm{SCN}}$ le rapport des concentrations en $\mathrm{SCN}^{-}$des phases organique et aqueuse à l'équilibre; il apparait (Fig. 1) que les courbes log $D_{\mathrm{SCN}}=f(\mathrm{pH})$ sont des droites de pentes voisines de -1 , ce qui montre que l'extraction des ions $\mathrm{SCN}^{-}$ se fait sous la forme d'acide thiocyanique HSCN associé au HDBPP. Les courbes $\log D_{\mathrm{SCN}}=f(\log -$ [HDBPP]) à divers $\mathrm{pH}$ constants (Fig. 2) sont des droites de pentes voisines de 0,7 , ce qui reste compatible avec l'équilibre d'extraction suivant:

$$
\mathrm{H}^{+}+\mathrm{SCN}^{-}+\overline{\mathrm{HDBPP}} \rightleftharpoons \overline{\mathrm{HSCN} . \mathrm{HDBPP}}
$$




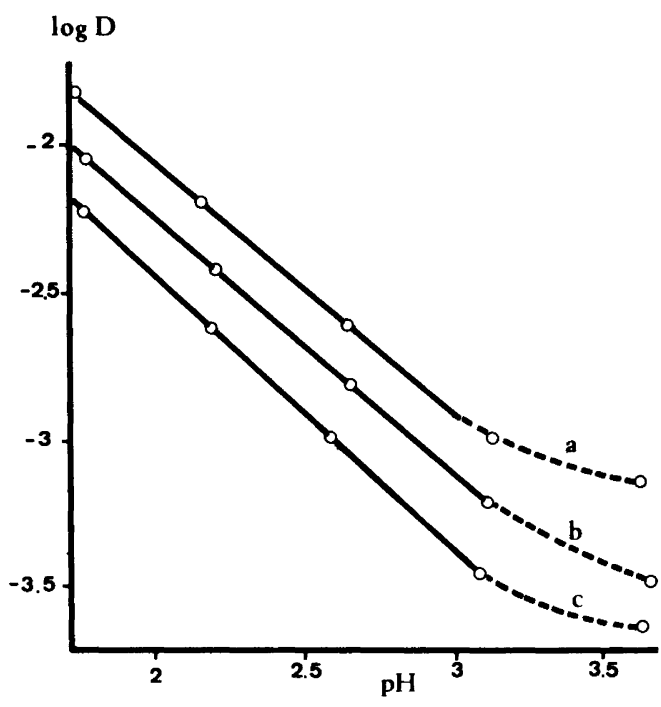

Fig. 1. Extraction de $\mathrm{SCN}^{-}$par le HDBPP: $\log$ $\mathrm{D}_{\mathrm{SCN}}=\mathrm{f}(\mathrm{pH}) .([\mathrm{KSCN}]=1 \mathrm{M} . \mathrm{a}:[\overline{\mathrm{HDBPP}}]=0,2$ $\mathrm{M} ;$ pente $=-0,85 . \mathrm{b}:[\overline{\mathrm{HDBPP}}]=0,1 \mathrm{M} ;$ pente $=$ $-0,85$. c: $[$ HDBPP $]=0,05 \mathrm{M}$; pente $=-0,95$ ).

La similitude des courbes d'extraction de l'acide thiocyanique par le HDBPP et par le TBP (Fig. 3) confirme l'équilibre d'extraction (4) proposé ci-

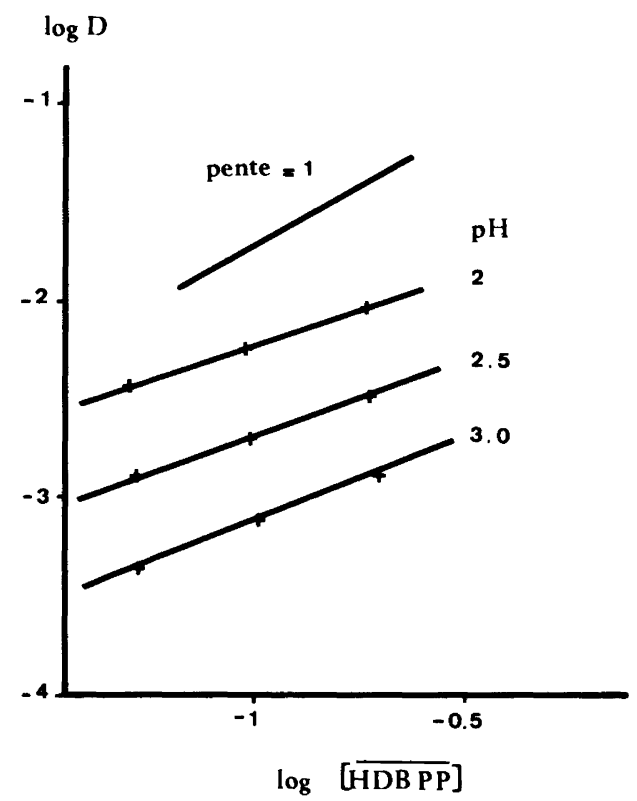

Fig. 2. Extraction de $\mathrm{SCN}^{-}$par le HDBPP: $\log D=f(\log [\overline{\mathrm{HDBPP}}])([\mathrm{KSCN}]=1 \mathrm{M})$.

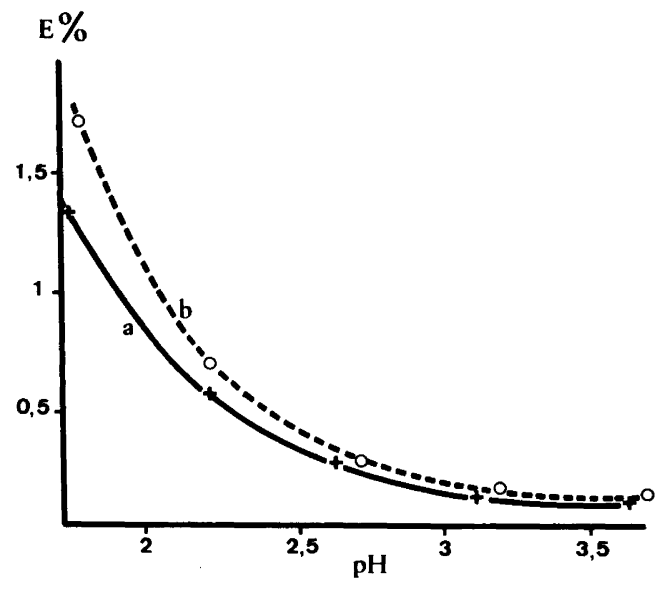

Fig. 3. Pourcentage de $\mathrm{SCN}^{-}$extrait (E \%) par le HDBPP et par le TBP. $([\mathrm{KSCN}]=1 \mathrm{M}$; a: $[\overline{\mathrm{HDBPP}}]=0,2 \mathrm{M} ; \mathrm{b}:[\mathrm{TBP}]=0,2 \mathrm{M}$ ) les volumes des deux phases étant égaux.

dessus: Khopkar et coll. ${ }^{11}$ ont en effet montré que l'acide thiocyanique est extrait par le TBP sous la forme $\overline{\mathrm{HSCN} . T B P}$. Ceci montre par ailleurs que c'est le groupement phosphoryle qui joue une rôle prépondérant lors de l'extraction, le groupement carbonyle n'ayant vraisemblablement qu'un effet secondaire. HDBPP et TBP ont également des pouvoirs extractants très voisins vis-à-vis des autres acides minéraux lesquels sont extraits sous la forme $\overline{\mathrm{HX} . \mathrm{S}}$ où $\mathrm{HX}=\mathrm{HCl}, \mathrm{HClO}_{4}$ ou $\mathrm{HNO}_{3}$ et $\mathrm{S}=\mathrm{TBP}$ ou HDBPP. ${ }^{18}$

Extraction du gallium et de l'indium

Influence $d u p H$. Au dessus de $\mathrm{pH} 2$, les extractions du gallium et de l'indium en milieu $\mathrm{SCN}^{-}$par le HDBPP dans le toluène sont indépendantes du $\mathrm{pH}$ de la solution aqueuse, les autres paramètres restant inchangés (Fig. 4); ceci montre que l'extraction se fait par simple addition. Si le $\mathrm{pH}$ devient inférieur à 2, l'extraction diminue ce qui s'explique par une extraction non négligeable d'acide thiocyanique (voir Fig. 3).

Influence de la concentration en $\mathrm{SCN}^{-}$. $\mathrm{Si}$ l'indium et le gallium sont extraits par le HDBPP sous la forme $\mathrm{M}(\mathrm{NCS})_{3}(\mathrm{HDBPP})_{x}$, pour une concentration donnée en HDBPP, l'extraction est alors proportionnelle à la fraction de complexe neutre $\mathbf{M}(\mathrm{NCS})_{3}$ présent en phase aqueuse. L'importance de l'extraction dépend alors directement des valeurs des constantes de formation des com- 


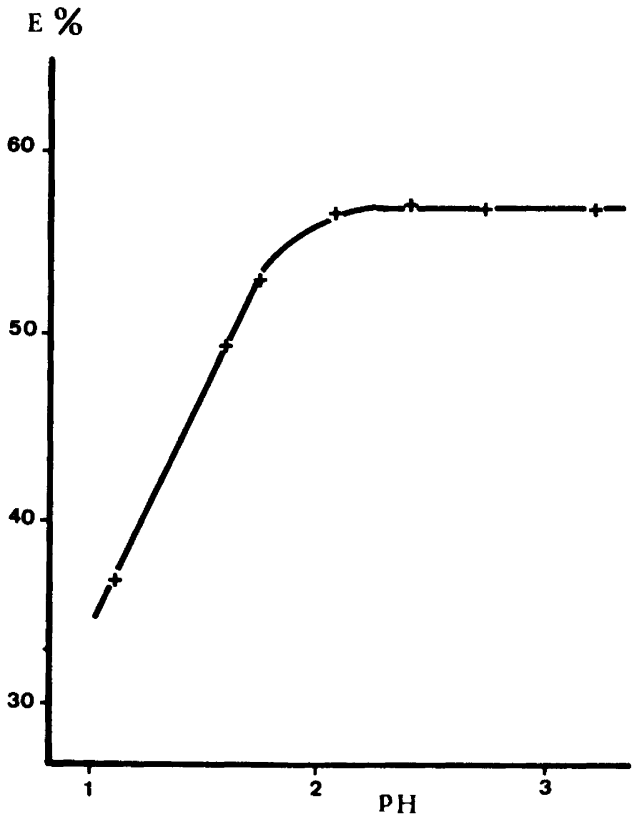

Fig. 4. Influence du pH sur le pourcentage d'indium extrait $(\mathrm{E} \%)$. ([KSCN] $=1 \mathrm{M}$; [HDBPP $]=0,05 \mathrm{M})$. les volumes des deux phases étant égaux.

plexes successifs $\mathrm{M}(\mathrm{NCS})_{n}^{3-n}$ et de la concentration en ions $\mathrm{SCN}^{-}$de la phase aqueuse.

Dans le cas de l'indium, l'extraction par le HDBPP passe par un maximum pour $\left[\mathrm{SCN}^{-}\right.$] $\sim 0,5 \mathrm{M}$ ce qui est en bon accord avec les valeurs des constantes $\beta_{n}$ (équation 1) déterminées par Hasegawa et Sekine. ${ }^{7}$ Pour $\left[\mathrm{SCN}^{-}\right]>0,5 \mathrm{M}$, la formation d'espèces anioniques $\mathrm{In}(\mathrm{NCS})_{3+n}^{n-}$ non extractibles, aux dépens de l'espèce extractible $\operatorname{In}(\mathrm{NCS})_{3}$, est responsable de la diminution de l'extraction.

En ce qui concerne le gallium, l'extraction croît régulièrement en fontion de la concentration croissante en ions $\mathrm{SCN}^{-}$dans de domaine étudié $\left(0 \leq\left[\mathrm{SCN}^{-}\right] \leq 2 \mathrm{M}\right)$, ceci est conforme au fait que les espèces cationiques ou neutres $\mathrm{Ga}(\mathrm{NCS})_{n}^{3-n}$ $(n \leq 3)$ prédominent en phase aqueuse dans les conditions expérimentales. ${ }^{12}$

Ainsi, le comportement différent du gallium et de l'indium vis-à-vis des ions $\mathrm{SCN}^{-}$en milieu aqueux est le facteur déterminant qui permet la séparation des deux métaux. Des faits similaires ont d'ailleurs pu être observés lors de l'étude de l'extraction du gallium et de l'indium en milieu thiocyanate par la trilaurylamine. ${ }^{13}$

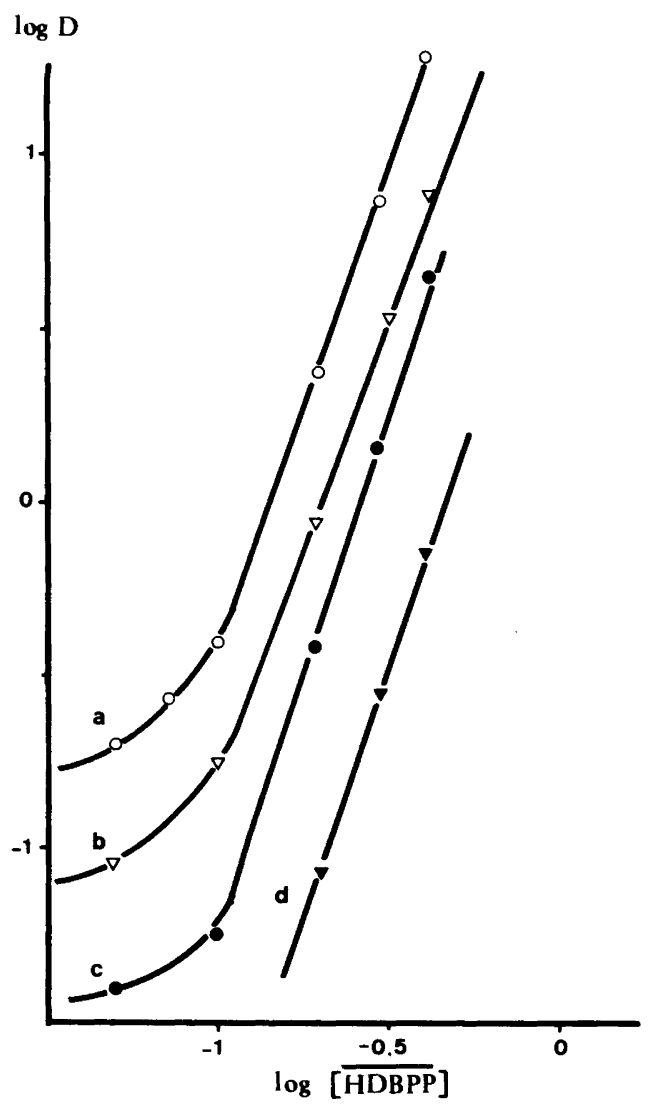

Fig. 5. Extraction du gallium. a: $[\mathrm{KSCN}]=2 \mathrm{M}$; pente $=2,7$. b: $[\mathrm{KSCN}]=1 \mathrm{M}$; pente $=2,75$. c: $[\mathrm{KSCN}]=0,5 \mathrm{M}$; pente $=3,0 . \mathrm{d}:[\mathrm{KSCN}]=0,2 \mathrm{M}$; pente $=3,0$.

Influence de la concentration en HDBPP. Dans le but de déterminer la composition des espèces extraites, nous avons étudié l'influence de la concentration en HDBPP sur les extractions du gallium et de l'indium. Les courbes $\log D=f(\log [\overline{\mathrm{HDBPP}}])$, obtenues sont reportées sur les Fig. 5 et 6 . Il s'agit de droites dont les pentes sont très voisines de 3 . Conformément à l'équation (3) et au raisonnement qui s'y rapporte, les espèces extraites peuvent s'écrire $\mathrm{Ga}(\mathrm{NCS})_{3}(\mathrm{HDBPP})_{3}$ et $\operatorname{In}(\mathrm{NCS})_{3}$ $(\mathrm{HDBPP})_{3}$.

Les coordinences du gallium et de l'indium étant au maximum de 6, le dibutylphénacylphosphonate ne peut-être coordiné sur ces métaux que par un seul atome d'oxygène donneur. Le caractère généralement plus donneur des groupements phos- 


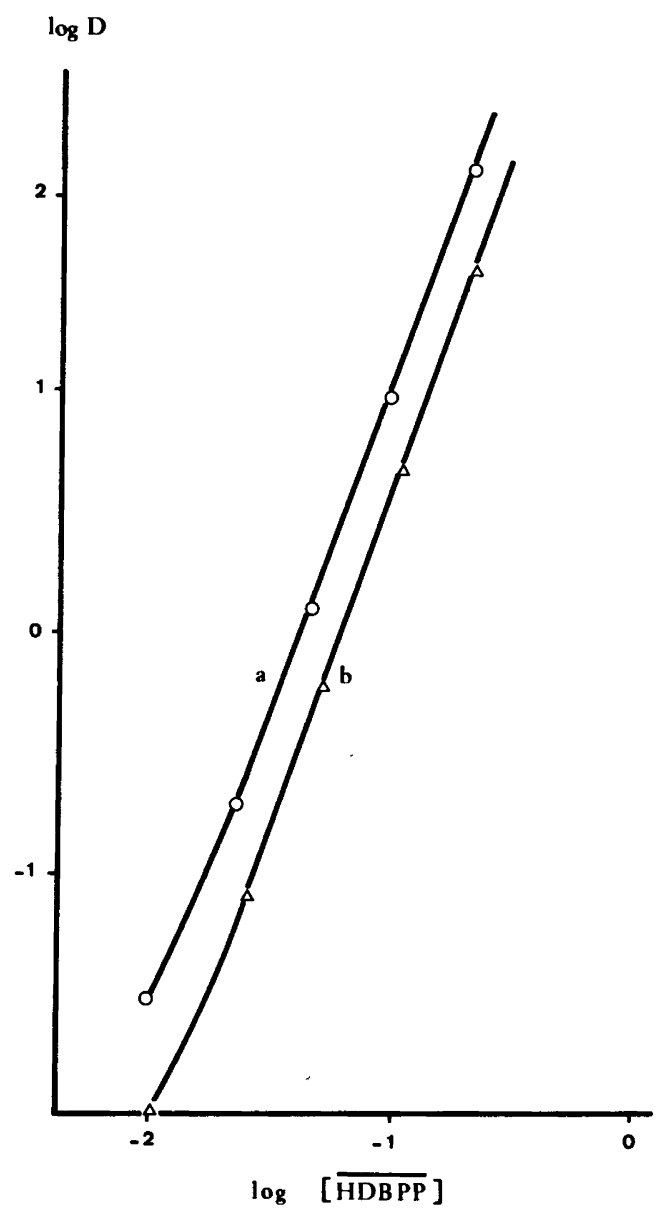

Fig. 6. Extraction de l'indium. a: $[\mathrm{KSCN}]=0,5 \mathrm{M}$; pente $=3,05$. b: $[\mathrm{KSCN}]=0,1 \mathrm{M}$, pente $=3,10$ ).

phoryles par rapport aux groupements carbonyles ainsi que les comportements très voisins du HDBPP et du TBP dans les mêmes conditions, laissent penser que le HDBPP joue ici un rôle d'extractant solvatant dont la fonction active est le groupement $P=O$. Une étude infrarouge a d'ailleurs montré qu'il en était ainsi dans le complexe $\mathrm{UO}_{2}\left(\mathrm{NO}_{3}\right)_{2}(\mathrm{HDBPP})_{2}$. Le HDBPP ne se coordine en fait par ses deux atomes d'oxygène que dans des conditions bien définies; c'est le cas, par exemple, lors de l'extraction acide de l'uranium qui ne peut être réalisée qu'à des pH élevés. ${ }^{18}$

Acta Chem. Scand. A 32 (1978) No. 5
E \%

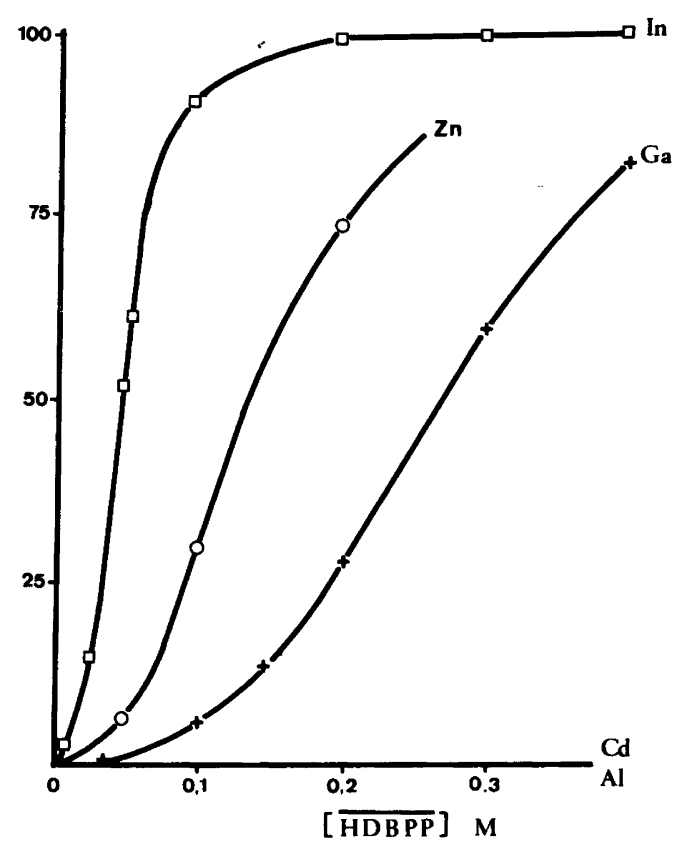

Fig. 7. Pourcentage d'extraction (E \%) de $\mathrm{Al}, \mathrm{Ga}$, $\mathrm{In}, \mathrm{Zn}$ et $\mathrm{Cd}$. [KSCN] $=0,5 \mathrm{M}$; valeur optimale permettant la séparation Ga-In, les volumes des deux phases étant égaux.

\section{Extraction d'autres métaux}

Extractions comparées des élements $\mathrm{Al}, \mathrm{Ga}, \mathrm{In}, \mathrm{Zn}$ et $C d$. La Fig. 7 représente les courbes d'extraction de ces métaux à partir de solutions aqueuses $0,5 \mathrm{M}$ en KSCN. Pour des concentrations en HDBPP comprises entre 0 et $0,4 \mathrm{M}$, cadmium et aluminium ne sont pas extraits ce qui peut permettre une séparation quantitative de l'indium de ces métaux en un seul équilibrage des phases organique et aqueuse: l'indium est en effect complètement extrait pour $[\overline{\mathrm{HDBPP}}] \geq 0,3 \mathrm{M}$. Zinc et gallium peuvent être séparés du cadmium et de l'aluminium, mais plusieurs étages d'extraction sont nécessaires. Dans une moindre mesure les séparations $\mathrm{In}-\mathrm{Ga}$ et In $-\mathrm{Zn}$ sont également possibles puisque pour $[\overline{\mathrm{HDBPP}}]=0,05 \mathrm{M}, 57 \%$ de l'indium est extrait contre $6 \%$ du zinc et $4 \%$ du gallium, et pour $[\overline{\mathrm{HDBPP}}]=0,1 \mathrm{M}, 91 \%$ de l'indium est extrait contre $5 \%$ du gallium.

Extraction du fer et du vanadium. Les Fig. 8 et 9 représentent les courbes d'extraction $\log D=f(\log$ 


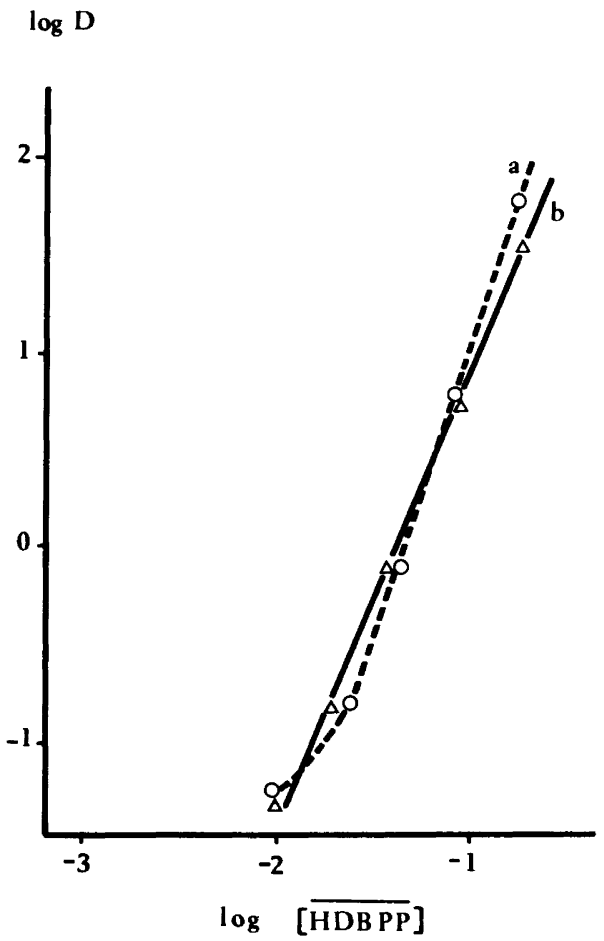

Fig. 8. Extraction du fer(III). a: $[\mathrm{KSCN}]=0,5 \mathrm{M}$; pente $=2,9 . \mathrm{b}:[\mathrm{KSCN}]=0,3 \mathrm{M} ;$ pente $=2,7$.

[ $\overline{\text { HDBPP }}])$ du fer(III) et du vanadium(IV) à diverses concentrations en KSCN. Dans tous les cas, il s'agit de droites dont les pentes sont voisines de 3, ce qui montre que les espèces extraites sont $\mathrm{Fe}(\mathrm{NCS})_{3}(\mathrm{HDBPP})_{3}$ et $\mathrm{VO}(\mathrm{NCS})_{2}(\mathrm{HDBPP})_{3}$. Là encore, compte tenu de la coordinence maximum de 6 de $\mathrm{Fe}$ (III) et de V(IV), le dibutylphenacylphosphonate n'est lié aux deux autres métaux que par l'intermédiaire de son groupement phosphoryle. Le HDBPP semble agir de la même façon vis-à-vis des terres rares; cependant, à des $\mathrm{pH}$ plus élevés $(\mathrm{pH} \geq 5)$, l'extraction prend un caractère acide marqué malgré la présence en solution aqueuse des ions $\mathrm{SCN}^{-}$; l'extraction fait alors vraisemblablement intervenir la forme anionique chélatante DBPP $^{-}$du HDBPP. ${ }^{18}$

Remerciements. Les auteurs tiennent à remercier la Centre National de la Recherche Scientifique, (Paris) pour son soutien financier: ce travail s'inscrit dans le cadre de l'Action Thématique Programmée «Epargne d'Energie et opérations chimiques industrielles».

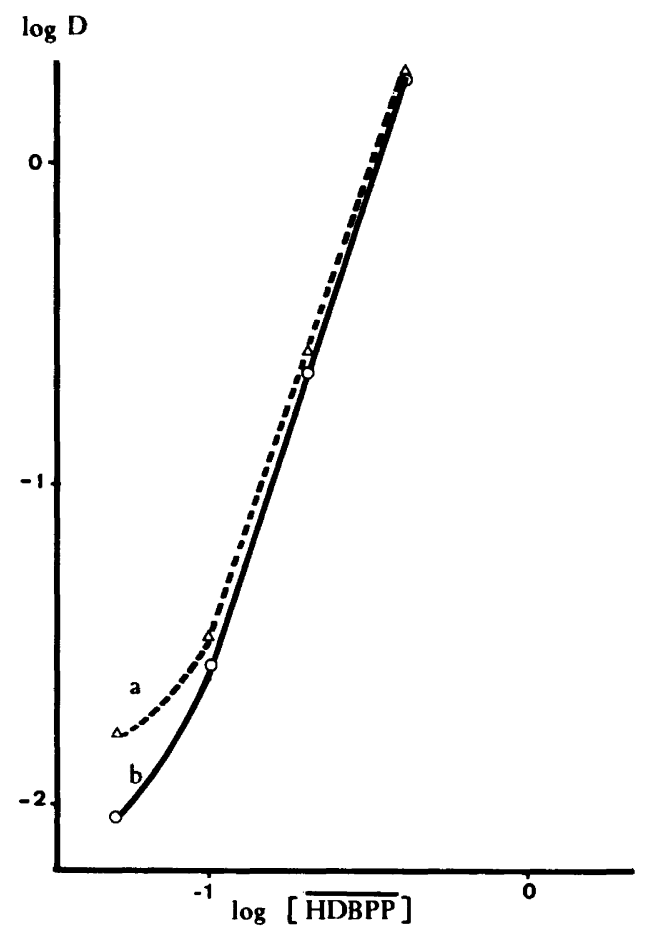

Fig. 9. Extraction du vanadium(IV). (a: $[\mathrm{KSCN}]=$ $0,5 \mathrm{M} ;$ pente $=2,98 . \mathrm{b}:[\mathrm{KSCN}]=2 \mathrm{M} ;$ pente $=3,03$ ).

\section{BIBLIOGRAPHIE}

1. Sekine, T. Bull. Chem. Soc. Jpn. 38 (1965) 1972.

2. Sekine, T. et Ishu, T. Bull. Chem. Soc. Jpn. 43 (1970) 2422.

3. Hasegawa, Y. Bull. Chem. Soc. Jpn. 43 (1970) 2665.

4. Sekine, T., Komatsy, Y. et Sakairi, M. Bull. Chem. Soc. Jpn. 44 (1971) 1480.

5. Moriya, M. et Sekine, T. Bull. Chem. Soc. Jpn. 44 (1971) 3347.

6. Hasegawa, Y., Takenchi, H. et Sekine, T. Bull. Chem. Soc. Jpn. 45 (1972) 1388.

7. Hasegawa, Y. et Sekine, T. J. Inorg. Nucl. Chem. 36 (1974) 421.

8. Specker, H. et Bankmann, E. Z. Anal. Chem. 149 (1956) 97.

9. Henning, K. et Specker, H. Z. Anal. Chem. 241 (1968) 81.

10. Golub, A. M., FamWang Ch'a et Samoilenko, V. M. Russ. J. Inorg. Chem. 16 (1971) 282.

11. Khopkar, P. K. et Narayanakkutty, P. J. Inorg. Nucl. Chem. 34 (1972) 2617.

12. Mikheev, N. B., Elfimova, G. J., Mikheeva, L. M. et Rumer, I. A. Vestnik. Mosk. Univ. Khim. 25 (1970) 742 . 
13. Brunette, J. P., Araujo-Barreira, M. L., Taheri, M. et Leroy, M. J. F. J. Inorg. Nucl. Chem. Accepté pour publication.

14. Gandon, L. et Goulaouic, M. French Pat. 2. 129.916 (1972).

15. Gaudernack, B., Hannestad, G. et Hundere, I. U.S. Pat. 3. 751.553 (1973).

16. Sevdic, D. et Meider-Gorican, H. J. Less. Common Metals 37 (1974) 103.

17. Bronzan, P. et Meider-Gorican, H. J. Less. Common Metals 29 (1972) 407.

18. Martin, J. L. et Leroy, M. J. F. (soumise a J. Chem. Res.).

Reçu le 28 Novembre 1977. 